\title{
STABILITY, NEIMARK-SACKER BIFURCATION AND CHAOS CONTROL FOR A PREY-PREDATOR SYSTEM WITH HARVESTING EFFECT ON PREDATOR
}

\author{
ÖZLEM AK GÜMÜŞ AND MICHAL FEČKAN
}

Received 23 September, 2020

\begin{abstract}
This paper deals with the dynamic behavior of a prey predator model obtained by the forward Euler method. We investigate the complex dynamics of discrete-time prey-predator system related to predator population which is subject to the effects of harvesting. The stability of equilibrium point of the model and also the existence and the direction of Neimark-Sacker bifurcation are analyzed. We show that the system undergoes Neimark-Sacker bifurcations by using center manifold theorem and bifurcation theory. A state feedback method is applied in order to control the Neimark-Sacker bifurcation. Moreover, numerical simulations are carried out to demonstrate the theoretical results obtained for stability, bifurcation and chaos control strategy.
\end{abstract}

2010 Mathematics Subject Classification: 37G35; 39A30; 39A33; 91B76; 92B05; 92D25

Keywords: prey-predator system, Neimark-Sacker bifurcation, stability, bifurcation theory, harvesting effect, chaos control

\section{INTRODUCTION}

Changes in population size result from interactions between species such as predatory, cooperative, mutualistic, commensal interactions. One of the fundamental structure in population dynamics is prey-predator interactions which has been described firstly by two pioneers Lotka-Volterra $[23,33]$. In recent times, many researchers have considered effects of harvesting on various types of prey-predator models $[2,10,16,24,25,34-36]$. Prey-predator harvesting which is applied on predator population as well as prey population have a strong impact on the dynamic evaluation [3,4]. Discrete-time and continuous-time models are used for predicting the size of a population. Discrete-time models are suitable for obtaining more accurate numerical simulations for non-overlapping generations [9, 11, 21, 22, 26]. Therefore, the studies on discretization of prey-predator models governed by difference

This work is partially supported by the Slovak Research and Development Agency under the contract No. APVV-18-0308 and by the Slovak Grant Agency VEGA No. 1/0358/20 and No. 2/0127/20. 
equations have received remarkable attention [15, 28, 29, 37, 38]. Bifurcation theory is widely used in the mathematical research of dynamic systems [12,20]. For the some type of bifurcation analysis of discrete-time population models, we refer to $[1,5,7,13-15,17,21,22,28,29,37,38]$. Dynamic models are also often used in chaos theory studies. A chaotic behavior occurs suddenly and includes unpredictable behavior. Chaos control theory offers some strategies avoid to the chaotic situation that occurs with these unpredictable behaviors [5-8, 19,27,30-32]. So, the chaotic behavior can be delayed or can be prevented completely. With this aspect, this theory is one of the important study subjects for dynamic systems. Lotka-Volterra model subject to harvesting effect on predator population is presented the following form:

$$
\begin{aligned}
& \frac{d x_{n}}{d t}=a x_{n}\left(1-x_{n}\right)-c x_{n} y_{n} \\
& \frac{d y_{n}}{d t}=b x_{n} y_{n}-e y_{n}-H y_{n} .
\end{aligned}
$$

where $x_{n}$ and $y_{n}$ denote the numbers of prey and predator, respectively. Moreover the parameters $a, b, c, e, H$ are positive and the initial conditions $x_{0}, y_{0}$ are positive real numbers.

In this paper, we will focus discrete-time version of the system (1.1) by applying the forward Euler scheme as follows:

$$
\begin{aligned}
& x_{n+1}=x_{n}+h\left[a x_{n}\left(1-x_{n}\right)-c x_{n} y_{n}\right] \\
& y_{n+1}=y_{n}+h\left[b x_{n} y_{n}-e y_{n}-H y_{n}\right]
\end{aligned}
$$

Here $H$ denotes harvesting effect. The important issues in discrete-time models to investigate the existence and the direction of the bifurcation and chaos phenomenon which are performed either by using numerical simulations or by using the center manifold theorem and bifurcation theory. Also, control of chaotic behavior in dynamic model using control strategies are remarkable .

Our purpose in this study is to obtain the equilibrium points of the system (1.2); and to investigate the stability conditions of these equilibrium points. Additionally, the direction and the existence of the Neimark-Sacker bifurcation of the system (1.2) is studied by choosing $b$ as the bifurcation parameter; and controlled the chaos behavior of the model via control strategy. Moreover, we show the dynamic properties of the system (1.2), by means of trajectories, bifurcation diagrams and phase portraits.

This study consists of four sections. In Section II, we investigate the existence and local asymptotic stability of the equilibrium points of the system (1.2) in $\mathbb{R}_{+}^{2}$. In Section III, we discuss the undergoing of the system (1.2) into a Neimark-Sacker bifurcation. In Section IV, we apply chaos control method to provide control of the system (1.2). Section V includes numerical simulations to support the theoretical results. In the last section, the results are briefly presented. 


\section{The Existence and Stability of Equilibrium Points}

In this section, we discuss the existence and stability of equilibrium points of the system (1.2) in the close first quadrant $\mathbb{R}_{2}^{+}$. A equilibrium point of (1.2) is a point $(\bar{x}, \bar{y})$ that satisfies

$$
\begin{aligned}
& \bar{x}=f(\bar{x}, \bar{y}), \\
& \bar{y}=g(\bar{x}, \bar{y}) .
\end{aligned}
$$

We easy obtain the equilibrium points of system (1.2) by using (2.1)

$$
\begin{aligned}
& \bar{x}=\bar{x}+h[a \bar{x}(1-\bar{x})-c \overline{x y}] \\
& \bar{y}=\bar{y}+h[b \overline{x y}-e \bar{y}-H \bar{y}] .
\end{aligned}
$$

It is clear that the system (1.2) has the equilibrium points $(0,0)$ and $(1,0)$ for all positive parameters. Additionaly, in the case of $b>e+H$, the system (1.2) has a unique positive coexistence equilibria $E_{2}=\left(\frac{e+H}{b}, \frac{a(b-e-H)}{b c}\right)$. So, we reach the results for existence of the equilibrium points of the system (1.2) as follows:

Lemma 1. For the system (1.2), the following cases hold:

(i) The system (1.2) has two equilibrium points. These are an trivial (extinction) equilibrium point $E_{0}=(0,0)$ and an exclusion (axial) equilibrium point $E_{1}=$ $(1,0)$ for all positive parameters;

(ii) If $b>e+H$, then the system (1.2) has a uniqe positive coexistence equilibrium point $E_{2}=\left(\frac{e+H}{b}, \frac{a(b-e-H)}{b c}\right)$.

The Jacobian matrix of a system (1.2) evaluated at any equilibrium point $(\bar{x}, \bar{y})$ is determined by

$$
J_{(\bar{x}, \bar{y})}=\left(\begin{array}{ll}
a_{11} & a_{12} \\
a_{21} & a_{22}
\end{array}\right)
$$

and the characteristic equation of matrix $J_{(\bar{x}, \bar{y})}$ can be given with

$$
\lambda^{2}-\operatorname{tr} J_{(\bar{x}, \bar{y})} \lambda+\operatorname{det} J_{(\bar{x}, \bar{y})}=0 .
$$

Suppose that $\lambda_{1}$ and $\lambda_{2}$ be two roots of $F(\lambda)=0$. Then the equilibrium point $(\bar{x}, \bar{y})$ is local asymptotic stable, if $\left|\lambda_{1}\right|<1$ and $\left|\lambda_{2}\right|<1$, and it is called as a sink point. Otherwise, $(\bar{x}, \bar{y})$ is always unstable, and it is known source (repeller). And, the equilibrium point $(\bar{x}, \bar{y})$ is called a saddle point if $\left|\lambda_{1}\right|<1$ and $\left|\lambda_{2}\right|>1$ (or $\left|\lambda_{1}\right|$ $>1$ and $\left|\lambda_{2}\right|<1$ ). If $\left|\lambda_{1}\right|=1$ or $\left|\lambda_{2}\right|=1$, then $(\bar{x}, \bar{y})$ is known as non-hyperboic point.

Now, let us study the local dynamics of the equilibrium points by obtaining the Jacobian matrix evaluated at $E_{0}, E_{1}$ and $E_{2}$. Firstly, by considering (1.2), we can get the Jacobian matrix as

$$
J_{E_{0}}=\left(\begin{array}{cc}
1+a h & 0 \\
0 & 1-e h-h H
\end{array}\right)
$$


evaluated at $E_{0}$, and the eigenvalues of $J_{E_{0}}$ are

$$
\lambda_{1}=1+a h, \quad \lambda_{2}=1-e h-h H .
$$

Thus, we can express the topological classification of the equilibrium point $E_{0}$.

Lemma 2. For the extinction equilibrium point $E_{0}$, the following cases hold:

(i) The equilibrium point $E_{0}$ cannot be a sink point. It is a source point for $e h+h H>2$.

(ii) If eh $+h H<2$, then the equilibrium point $E_{0}$ is a saddle point.

Secondly, we can get the Jacobian matrix evaluated at $E_{1}$ as

$$
J_{E_{1}}=\left(\begin{array}{cc}
1-a h & -c h \\
0 & 1+b h-e h-h H
\end{array}\right)
$$

and the eigenvalues of $J_{E_{1}}$ are

$$
\lambda_{1}=1-a h, \quad \lambda_{2}=1+b h-e h-h H .
$$

From there, we can get the topological classification of the equilibrium point $E_{1}$.

Lemma 3. For the extinction equilibrium point $E_{1}$, the following cases hold:

(i) If $a h<2$ and $(b<e+H$ and $(e+H-b) h<2)$, then the equilibrium point $E_{1}$ is a sink point.

(ii) If $a h<2$ and $(b>e+H$ or $(H+e-b) h>2)$ or $a h>2$ and $(b<e+H$ and $(e+H-b) h<2)$, then the equilibrium point $E_{1}$ is a saddle point.

(iii) If $a h>2$ and $(b>e+H$ or $(H+e-b) h>2)$, then the equilibrium point $E_{1}$ is a source point.

In order to investigate the dynamics of a unique positive equilibrium point $E_{2}$, we need the following lemma.

Lemma 4. [22] Let $F(\lambda)=\lambda^{2}-\operatorname{trJ} \lambda+\operatorname{det} J$. Suppose that $F(1)>0$, and $\lambda_{1}$ and $\lambda_{2}$ are two roots of $F(\lambda)=0$. Then,

(i) $\left|\lambda_{1}\right|<1$ and $\left|\lambda_{2}\right|<1$ if and only if $F(-1)>0$ and $\operatorname{det} J<1$;

(ii) $\left|\lambda_{1}\right|<1$ and $\left|\lambda_{2}\right|>1$ (or $\left|\lambda_{1}\right|>1$ and $\left|\lambda_{2}\right|<1$ ) if and only if $F(-1)<0$;

(iii) $\left|\lambda_{1}\right|>1$ and $\left|\lambda_{2}\right|>1$ if and only if $F(-1)>0$ and $\operatorname{det} J>1$;

(iv) $\lambda_{1}=-1$ and $\left|\lambda_{2}\right| \neq 1$ if and only if $F(-1)=0$ and $-\operatorname{trJ} \neq 0,2$;

(v) $\lambda_{1}$ and $\lambda_{2}$ are complex and $\left|\lambda_{1}\right|=\left|\lambda_{2}\right|=1$ if and only if $(-t r J)^{2}-4 \operatorname{det} J<0$ and $\operatorname{det} J=1$.

At last, we can give the Jacobian matrix as

$$
J_{E_{2}}=\left(\begin{array}{cc}
\frac{b-a h(e+H)}{b} & -\frac{c h(e+H)}{b} \\
\frac{a h(b-e-H)}{c} & 1
\end{array}\right)
$$


evaluated at $E_{2}$, and the characteristic polynomial of $J_{E_{2}}$ is

$$
F(\lambda)=\lambda^{2}-\left[1+\frac{b-a h(e+H)}{b}\right] \lambda+\left[\frac{b-a h(e+H)}{b}+\frac{a c h^{2}(b-e-H)(e+H)}{b c}\right] .
$$

By using Lemma 4, we can get the topological classification of the coexistence positive equilibrium $E_{2}$.

Lemma 5. For the coexistence positive equilibrium point $E_{2}$, the following cases hold:

(i) If

$$
\frac{2 a e h+a e^{2} h^{2}+2 a h H+2 a e h^{2}+a h^{2} H^{2}}{4+a e h^{2}+a h^{2} H}<b<\frac{1+e h+h H}{h},
$$

then the equilibrium point $E_{2}$ is a sink point.

(ii) If

$$
b>\frac{1+e h+h H}{h},
$$

then the equilibrium point $E_{2}$ is a source point.

(iii) If

$$
b<\frac{2 a e h+a e^{2} h^{2}+2 a h H+2 a e h^{2}+a h^{2} H^{2}}{4+a e h^{2}+a h^{2} H},
$$

then the equilibrium point $E_{2}$ is a saddle point.

(iv) If

$$
b=\frac{2 a e h+a e^{2} h^{2}+2 a h H+2 a e h^{2}+a h^{2} H^{2}}{4+a e h^{2}+a h^{2} H}
$$

or

$$
b=\frac{1+e h+h H}{h},
$$

then the equilibrium point $E_{2}$ is non-hyperbolic point.

Example 1. For the parameter values $a=2.5, b=2.25, e=0.2, c=1, H=1$ and $h=0.9$ the initial conditons $\left(x_{0}, y_{0}\right)=(0.3,0.4)$, the equilibrium point $E_{2}$ of the model (1.2) is locally asymptotically stable. Moreover, for $b=2.31$, the equilibrium point $E_{2}$ of the model (1.2) is non-hyperbolic point. To confirm the theoretical result, the trajectories of the model (1.2) are shown in Figure 1.

\section{NEIMARK-SACKER BIFURCATIONS}

In this section, the direction and existence of Neimark-Sacker bifurcation are obtained for the system (1.2). Additionally, if the system (1.2) provides eigenvalue assignment, transversality and non resonance conditions, then a Neimark-Sacker bifurcation occurs at a bifurcation point $[12,30]$. Considering Lemma 4-(v), the bifurcation occurs in non-hyperbolic point $E_{2}$ where the roots of $J_{E_{2}}$ are complex conjugate with module one. In order to work Neimark-Sacker bifurcation in the system (1.2), 

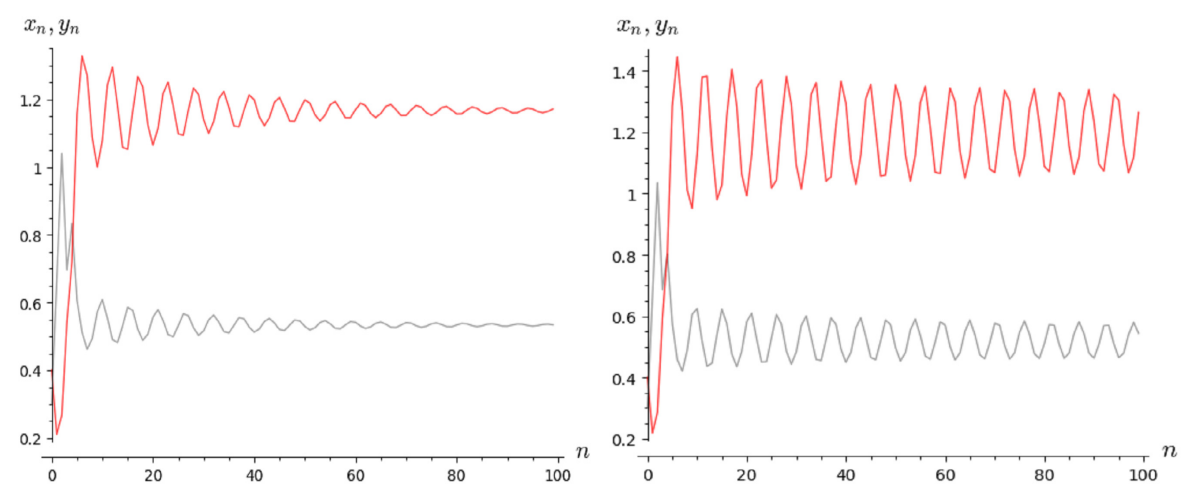

FIGURE 1. (a) Trajectories of the prey-predator system (1.2) with the parameter values $a=2.5, b=2.25, e=0.2, c=1, H=1$ and $h=0.9$ the initial conditions $\left(x_{0}, y_{0}\right)=(0.3,0.4)$. (b) Trajectories of the prey-predator system (1.2) with the parameter values $a=2.5, b=2.31, e=0.2, c=1, H=1$ and $h=0.9$ the initial conditions $\left(x_{0}, y_{0}\right)=(0.3,0.4)$.

we define the parameters providing non-hyperbolic conditions by taking the bifurcation parameter $b$ as follows:

$$
N S B_{E_{2}}=\left\{\begin{array}{c}
a, b, c, e h, H \varepsilon \mathbb{R}^{+}: b>\frac{e+H}{2}+\frac{1}{2} \sqrt{a e+e^{2}+(a+2 e) H+H^{2}} \\
\text { and } b=b_{1}=\frac{1+e h+h H}{h}
\end{array}\right\} .
$$

The eigenvalues of $J_{E_{2}}$ have a pair of conjugate complex numbers which are of module one for $b=b_{1}$. These eigenvalues are found as

$$
\lambda, \bar{\lambda}=\left.\frac{\operatorname{tr} J_{E_{2}}}{2} \mp \frac{\sqrt{4 \operatorname{det} J_{E_{2}}-\left(t r J_{E_{2}}\right)^{2}}}{2}\right|_{b=b_{1}}
$$

where

$$
\lambda, \bar{\lambda}=\left.\frac{1}{2 b}\left[2 b-a e h-a h H \mp i h \sqrt{a(e+H)\left[4 b^{2}-a e-4 b e-a H-4 b H\right]}\right]\right|_{b=b_{1}}
$$

such that

$$
|\lambda|=|\bar{\lambda}|=1
$$

For $b \in N S B_{E_{2}}$, we get

$$
\left.\frac{\partial\left|\lambda_{i}(b)\right|}{\partial b}\right|_{b=b_{1}} \neq 0, \quad i=1,2 .
$$

Additionally, if

$$
\left.\operatorname{trJ}_{E_{2}}\right|_{b=b_{1}} \neq 0,-1
$$


then, we reach the following:

$$
\lambda^{k}\left(b_{1}\right) \neq 1, \quad k=1,2,3,4 .
$$

Here, the value $\operatorname{tr} J_{E_{2}}$ at the point $b=b_{1}$ is

$$
\operatorname{trJ}_{E_{2}}=\frac{2 e H-a h^{2}(e+H)+2(1+h H)}{1+e h+h H} .
$$

Moreover, the eigenvalues of $J_{E_{2}}$ evaluated at the point $b=b_{1}$ are obtained as

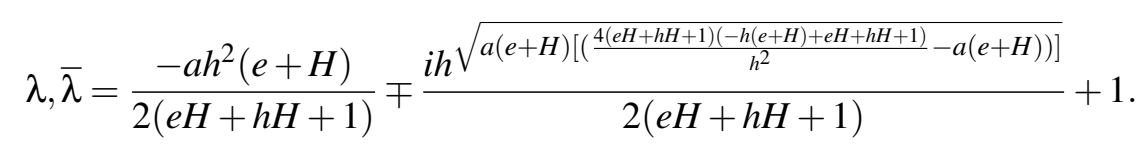

and

$$
\begin{aligned}
& \left.\frac{\partial\left|\lambda_{i}(b)\right|}{\partial b}\right|_{b=b_{1}}= \\
& \frac{a h^{2}(e+H) h \sqrt{a(e+H) \frac{4(e H+h H+1)(-h(e+H)+e H+h H+1)}{h^{2}}}}{\left(2(e H+h H+1)^{2} \sqrt{a(e+H) \frac{4(e H+h H+1)(-h(e+H)+e H+h H+1)}{h^{2}}}\right)-a(e+H)} \\
& \frac{-a(e+H)-i a h(e+H)-2 i(e+H)(e H+h H+1)}{2(e H+h H+1)^{2} \sqrt{a(e+H) \frac{4(e H+h H+1)(-h(e+H)+e H+h H+1)}{h^{2}}}-a(e+H)} .
\end{aligned}
$$

Let $q, p \in \mathbb{C}^{2}$ be two eigenvectors which correspond to the eigenvalues $\lambda$ of the matrix $J\left(\Omega_{N S B_{E 2}}\right)$ and the eigenvalues $\bar{\lambda}$ of the matrix $J\left(\Omega_{N S B_{E 2}}\right)^{T}$, respectively. These eigenvectors are calculated as

$$
q \sim\left(\frac{-1-\frac{1}{2}\left[-1-M-i \sqrt{2 M-1-M^{2}-4 N S}\right]}{S}, 1\right)
$$

and

$$
p \sim\left(\frac{-1-\frac{1}{2}\left[-1+M+i \sqrt{2 M-1-M^{2}-4 N S}\right]}{N}, 1\right)
$$

such that

$$
\begin{aligned}
M & =\frac{1+e H-a h^{2}(e+H)+h H}{1+e H+h H}, \\
N & =-\frac{c h^{2}(e+H)}{1+e H+h H} \\
& \text { and } \\
S & =\frac{a(1+e H-e h)}{c} .
\end{aligned}
$$


By using the scalar product in $\mathbb{C}^{2}:\langle p, q\rangle=\overline{p_{1}} q_{1}+\overline{p_{2}} q_{2}$, we define the following vector in order to normalize $p$ according to $q$

$$
p \sim\left(\frac{(M-1)-i \sqrt{2 M-1-M^{2}-4 N S}}{R^{*}}, \frac{1}{R^{*}}\right)
$$

where $R^{*}=\frac{(M+1)+i \sqrt{2 M-1-M^{2}-4 N S}}{4 N S}+1$ such that $\langle p, q\rangle=1$.

In order to translate the equilibrium point $E_{2}$ of the system (1.2) into the origin $(0,0)$, we take

$$
u_{n}=x_{n}-\frac{e+H}{b}, v_{n}=y_{n}-\frac{a(b-e-H)}{b c} .
$$

Then, we get the following map:

$$
\left(\begin{array}{c}
u \\
v
\end{array}\right) \rightarrow J_{E_{2}}\left(\begin{array}{c}
u \\
v
\end{array}\right)+\left(\begin{array}{c}
F_{1}(u, v) \\
F_{2}(u, v)
\end{array}\right)
$$

where

$$
\begin{aligned}
& F_{1}(u, v)=-c h u v-a h u^{2}+O\left(\|U\|^{3}\right) \\
& F_{2}(u, v)=b h u v+O\left(\|U\|^{3}\right) .
\end{aligned}
$$

such that $U_{t}=(u, v)^{T}$. Additionally, the system (1.2) can be written as

$$
\left(\begin{array}{c}
u_{n+1} \\
v_{n+1}
\end{array}\right) \rightarrow J_{E_{2}}\left(\begin{array}{c}
u_{n} \\
v_{n}
\end{array}\right)+\frac{1}{2} B\left(u_{n}, v_{n}\right)+\frac{1}{6} C\left(u_{n}, v_{n}, w_{n}\right)+O\left(\left\|U_{n}\right\|^{4}\right),
$$

with the multi-linear vector functions of $u ; v ; w \in \mathbb{R}^{2}$ :

$$
B(u, v)=\left(\begin{array}{c}
B_{1}(u, v) \\
B_{2}(u, v)
\end{array}\right)
$$

and

$$
C(u, v)=\left(\begin{array}{c}
B_{1}(u, v, w) \\
B_{2}(u, v, w)
\end{array}\right) .
$$

These vectors are expressed by

$$
\begin{aligned}
B_{1}(u, v) & =\left.\sum_{j, k=1}^{2} \frac{\partial^{2} F_{1}}{\partial \xi_{j} \partial \xi_{k}}\right|_{\xi=0} u_{j} v_{k}=-2 a h u_{1} v_{1}-\operatorname{ch}\left(u_{2} v_{1}+u_{1} v_{2}\right) \\
B_{2}(u, v) & =\left.\sum_{j, k=1}^{2} \frac{\partial^{2} F_{2}}{\partial \xi_{j} \partial \xi_{k}}\right|_{\xi=0} u_{j} v_{k}=b h\left(u_{2} v_{1}+u_{1} v_{2}\right), \\
C_{1}(u, v, w) & =\left.\sum_{j, k=1}^{2} \frac{\partial^{3} F_{1}}{\partial \xi_{j} \partial \xi_{k} \xi_{l}}\right|_{\xi=0} u_{j} v_{k} w_{l}=0 \\
C_{2}(u, v, w) & =\left.\sum_{j, k=1}^{2} \frac{\partial^{3} F_{2}}{\partial \xi_{j} \partial \xi_{k} \xi_{l}}\right|_{\xi=0} u_{j} v_{k} w_{l}=0 .
\end{aligned}
$$


$\forall U \in \mathbb{R}^{2}$ can be uniquely shown as

$$
U=z q+\overline{z q}
$$

for some $z \in \mathbb{C}$. Here, $\bar{z}$ is the conjugate of the complex number $z$, and $z=\langle p, U\rangle$. For all sufficiently small $|b|$, we can transform the system (1.2) into the form,

$$
z \rightarrow \lambda(b) z+g(z, \bar{z}, b)
$$

where $\lambda(r)=(1+\omega(b)) e^{i \arctan (b)}$ with $\omega\left(b_{1}\right)=0$ and $g(z, \bar{z}, b)$ is a complex valued smooth function of $z$ and $\bar{z}$. The Taylor expression of $g$ with respect to $g(z, \bar{z})$ is as follows:

$$
g(z, \bar{z}, b)=\sum_{k+l \geq 2} \frac{1}{k ! l !} g_{k l}(b) z^{k} \bar{z}^{l}
$$

with

$$
\begin{aligned}
& g_{20}\left(b_{1}\right)=<p, B(q, q)> \\
& g_{11}\left(b_{1}\right)=<p, B(q, \bar{q})> \\
& g_{02}\left(b_{1}\right)=<p, B(\bar{q}, \bar{q})> \\
& g_{21}\left(b_{1}\right)=<p, C(q, q, \bar{q})>.
\end{aligned}
$$

In order to come out as the Neimark-Sacker bifurcation for the system (3.11), we need that the coefficient $\varphi\left(b_{1}\right)$ must not be zero. This coefficient is

$$
\begin{aligned}
\varphi\left(b_{1}\right) & =\operatorname{Re}\left(\frac{e^{-i \arctan \left(b_{1}\right)}}{2} g_{21}\right)-\operatorname{Re}\left(\frac{\left(1-2 e^{i \arctan \left(b_{1}\right)}\right) e^{-2 i \arctan \left(b_{1}\right)}}{2\left(1-e^{i \arctan \left(b_{1}\right)}\right)} g_{20} g_{11}\right) \\
& -\frac{1}{2}\left|g_{11}\right|^{2}-\frac{1}{4}\left|g_{02}\right|^{2}
\end{aligned}
$$

where $e^{i \arctan \left(b_{1}\right)}=\lambda\left(b_{1}\right)$. Consequently, we have the following theorem on NeimarkSacker bifurcation:

Theorem 1. If (3.5) holds, $\varphi\left(b_{1}\right) \neq 0$ and the parameter $b$ changes its value in a small vicinity of $N S B_{E 2}$, then the system (1.2) passes through a Neimark-Sacker bifurcation at the only equilibrium point $E_{2}$. Moreover, if $\varphi\left(b_{1}\right)<0\left(\varphi\left(b_{1}\right)>0\right)$, then there exists a unique attracting (repelling) invariant closed curve which bifurcates from $E_{2}$.

\section{Chaos Control}

Stabilizing unstable periodic orbits in chaotic dynamics has been an interesting research topic in recent years [5-7]. Chaos can be controlled by various methods (see $[8,19,27,31,37])$. The first feedback control strategy known as the OGY method was proposed by Ott et al. [27]. We study to control the chaos via chaos controlling strategy based a state feedback control method $[8,19]$. Now, we suppose that system 
(1.2) undergoes Neimark-Sacker bifurcation at equilibrium point $(\bar{x}, \bar{y})$, then corresponding controlled system can be taken as follows:

$$
\begin{aligned}
& x_{n+1}=x_{n}+h\left(a x_{n}\left(1-x_{n}\right)-c x_{n} y_{n}\right)-k_{1}\left(x_{n}-\bar{x}\right)-k_{2}\left(y_{n}-\bar{y}\right) \\
& y_{n+1}=y_{n}+h\left[\left(b x_{n} y_{n}-e y_{n}-H y_{n}\right]\right.
\end{aligned}
$$

such that $-k_{1}\left(x_{n}-\bar{x}\right)-k_{2}\left(y_{n}-\bar{y}\right)$ is the control force; and $k_{1}$ and $k_{2}$ define as the feedback gains. The Jacobian matrix of $J(\bar{x}, \bar{y})$ of controlled system (4.1) can be written as

$$
J(\bar{x}, \bar{y})=\left[\begin{array}{cc}
\frac{b-a h(e+H)-b k_{1}}{b} & \frac{-c h(e+H)+b k_{2}}{b} \\
\frac{a h(b-e-H)}{c} & 1
\end{array}\right] .
$$

The characteristic equation of the Jacobian matrix $J(\bar{x}, \bar{y})$ is given by

$$
\begin{gathered}
\mu^{2}-\frac{2 b-a h(e+H)-b k_{1}}{b} \mu \\
+\frac{c\left(-a h(e+H)(1+e h+h H)+b\left(1+a h^{2}(e+H)\right)-b c k_{1}+a b h(b-e-h) k_{2}\right.}{b c}=0 .
\end{gathered}
$$

Let $\mu_{1}$ and $\mu_{2}$ be the roots of characteristic equation system (4.2), then we have

$$
\begin{gathered}
\mu_{1}+\mu_{2}=\frac{2 b-a h(e+H)-b k_{1}}{b} \\
\mu_{1} \mu_{2}=\frac{c\left(-a h(e+H)(1+e h+h H)+b\left(1+a h^{2}(e+H)\right)-b c k_{1}\right.}{b c} \\
+\frac{a b h(b-e-h) k_{2}}{b c} .
\end{gathered}
$$

To determine the marginal stability lines, we must find the solution of the equations $\mu_{1}= \pm 1$ and $\mu_{1} \mu_{2}=1$. These conditions confirm that $\mu_{1}$ and $\mu_{2}$ have absolute value less than 1. Let us take $\mu_{1} \mu_{2}=1$, then from Eq. (4.4), we get

$$
l_{1}:=\frac{b c k_{1}+a h\left(c(e+H)\left(1+h(-b+e+H)+b(-b+e+H) k_{2}\right)\right.}{b c}=0 .
$$

Now, we suppose that $\mu_{1}=1$, then Eq. (4.3) and Eq. (4.4) imply

$$
\begin{aligned}
l_{2} & :=1+\frac{c\left(-a h(e+H)(2+h(e+H))+b\left(4+a h^{2}(e+H)\right)-2 b k_{1}\right)}{b c} \\
& +\frac{a b h(b-e-H) k_{2}}{b c}-\frac{-2 b+a h(e+H)+b k_{1}}{h}=0 .
\end{aligned}
$$

Moreover, from $\mu_{1}=-1$, by using Eq. (4.3) and Eq. (4.4), we obtain

$$
\begin{aligned}
l_{3} & :=1+\frac{c\left(-a h(e+H)(2+h(e+H))+b\left(4+a h^{2}(e+H)\right)-2 b k_{1}\right)}{b c} \\
& +\frac{a b h(b-e-H) k_{2}}{b c}+\frac{-2 b+a h(e+H)+b k_{1}}{h}=0 .
\end{aligned}
$$


The triangular region determined by the lines $l_{1}, l_{2}$ and $l_{3}$ in $k_{1} k_{2}$ plane is the region of the values that make the eigenvalues less than 1 .

\section{NumeriCAL Simulations}

In this section, theoretical results are supported with graphics by using Maple [32] and SageMath [18] programming. Some numerical simulations are given to demonstrate the existence of Neimark-Sacker bifurcation for the system (1.2). Here, bifurcation diagrams and phase portraits are illustrated by taking $b$ as the bifurcation parameter.

Example 2. Let us consider the following system for the parameter values $e=$ $0.2, a=2.5, c=1, H=1, h=0.9$ and $b=2.3111$,

$$
\begin{aligned}
& x_{n+1}=x_{n}+2.25 x_{n}\left(1-x_{n}\right)-0.9 x_{n} y_{n} \\
& y_{n+1}=y_{n}+2.07999 x_{n} y_{n}-0.18 y_{n}-0.9 y_{n} .
\end{aligned}
$$

The coexistence positive equilibrium point is $(\bar{x}, \bar{y})=(0.519233,1.20192)$, and the Jacobian matrix evaluated $(\bar{x}, \bar{y})$ is as follows:

$$
J_{(\bar{x}, \bar{y})}=\left(\begin{array}{cc}
-0.168276 & -0.46731 \\
2.49998 & 1
\end{array}\right) .
$$

If the eigenvalues are calculated, then we obtain

$$
\lambda_{1,2}=0.415862 \mp 0.909422 i
$$

such that $\left|\lambda_{1,2}\right|=1$. Let the complex eigenvectors $q, p \in \mathbb{C}^{2}$ be correspond to $\lambda_{1,2}$, respectively.

$$
q \sim(-0.3637718555-0.233656893 i, i)^{T}
$$

and

$$
p \sim(-0.3637718555+0.233656893 i,-i)^{T}
$$

If the normalized vector is taken as

$$
q \sim(-0.3637718555-0.233656983 i, i)^{T}
$$

to obtain the normalization $\langle p, q\rangle=1$, the vector is found as

$$
p \sim(0.336308-0.315341 i, 0.193293+1.04866 i)^{T} .
$$

By transformation of variables

$$
u_{n}=x_{n}-0.519233, v_{n}=y_{n}-1.20192
$$

the system (5.1) can be written as follows:

$$
\begin{aligned}
& u_{n+1}=-0.168277 u_{n}-0.46731 v_{n}-0.9 u_{n} v_{n}-2.25 u_{n}^{2} \\
& y_{n+1}=2.49998 u_{n}+v_{n}+2.07999 u_{n} v_{n} .
\end{aligned}
$$


When the coefficients of the form (3.15) are calculated, we get

$$
\begin{aligned}
& g_{20}\left(b_{1}\right)=-1.62334-1.59177 i \\
& g_{11}\left(b_{1}\right)=-0.313611+0.901413 i \\
& g_{02}\left(b_{1}\right)=1.48092-0.932656 i \\
& g_{21}\left(b_{1}\right)=0 .
\end{aligned}
$$

Here, $\theta=1.141903497$; and from (3.16), we get $\varphi\left(b_{N S}\right)=0.606259>0$. Consequently, a sub critical Neimark-Sacker bifurcation emerges at $b_{N S}=2.3111$.

To confirm the theoretical result, bifurcation diagrams and phase portraits of the prey-predator system (1.2) are shown in Figures 1-3 where the initial point is $\left(x_{0}, y_{0}\right)=$ $(0.3,0.4)$. From Figure 2(a) and Figure 2(b), we see that the equilibrium point $(\bar{x}, \bar{y})$
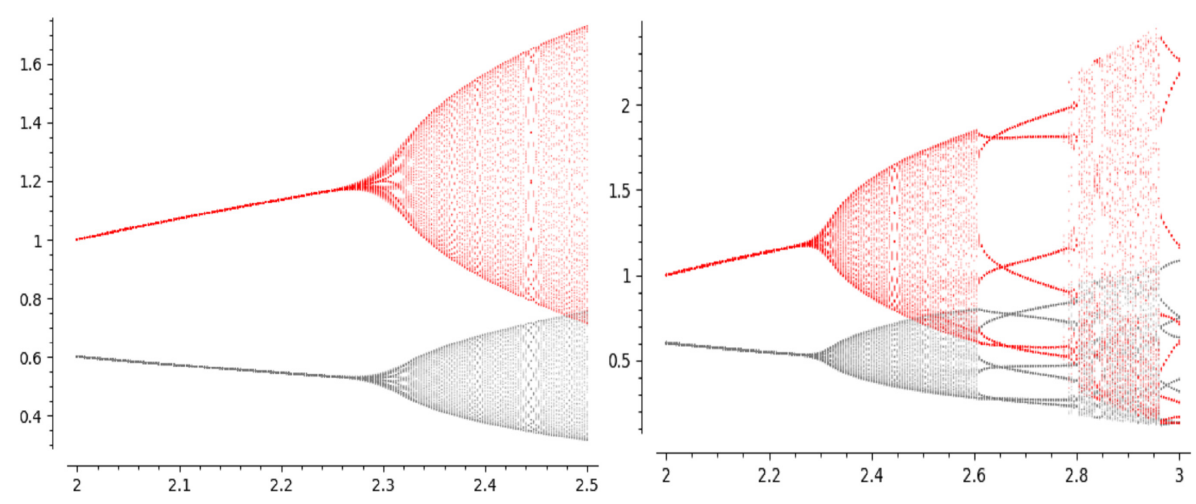

FIgURE 2. (a) Bifurcations Diagrams of the prey-predator system (1.2) for different values $b \in(2,2.5)$ with the parameter values $a=2.5, e=0.2, c=1, H=1$ and $h=0.9$ the initial conditions $\left(x_{0}, y_{0}\right)=(0.3,0.4)$. (b) Bifurcations Diagrams of the prey-predator system (1.2) for different values $b \in(2,3)$ with the parameter values $a=2.5, e=0.2, c=1, H=1$ and $h=0.9$ the initial conditions $\left(x_{0}, y_{0}\right)=(0.3,0.4)$.

of the system (1.2) is stable for $b<2.3111$ and loses its stability at $b=2.3111$, and close invariant curves appear around the positive equilibrium point if $b>2.3111$. More clearly, when the parameter $b$ exceeds 2.3111 , the equilibrium point is unstable and a repelling invariant closed curve bifurcates from the positive equilibrium point. The phase portaits of bifurcation diagrams in Figure 2(a) and 2(b) for different values of $b$ are shown in Figure 3, which clearly demonstrates the process how smooth invariant curve bifurcates from the stable equilibrium point and increases its radius.

Example 3. We consider system (1.2) with the parameter values $a=2.5, e=0.2$, $c=1, H=1, h=0.9$, and initial condition $\left(x_{0}, y_{0}\right)=(0.3,0.4)$. When $2 \leq b<$ 

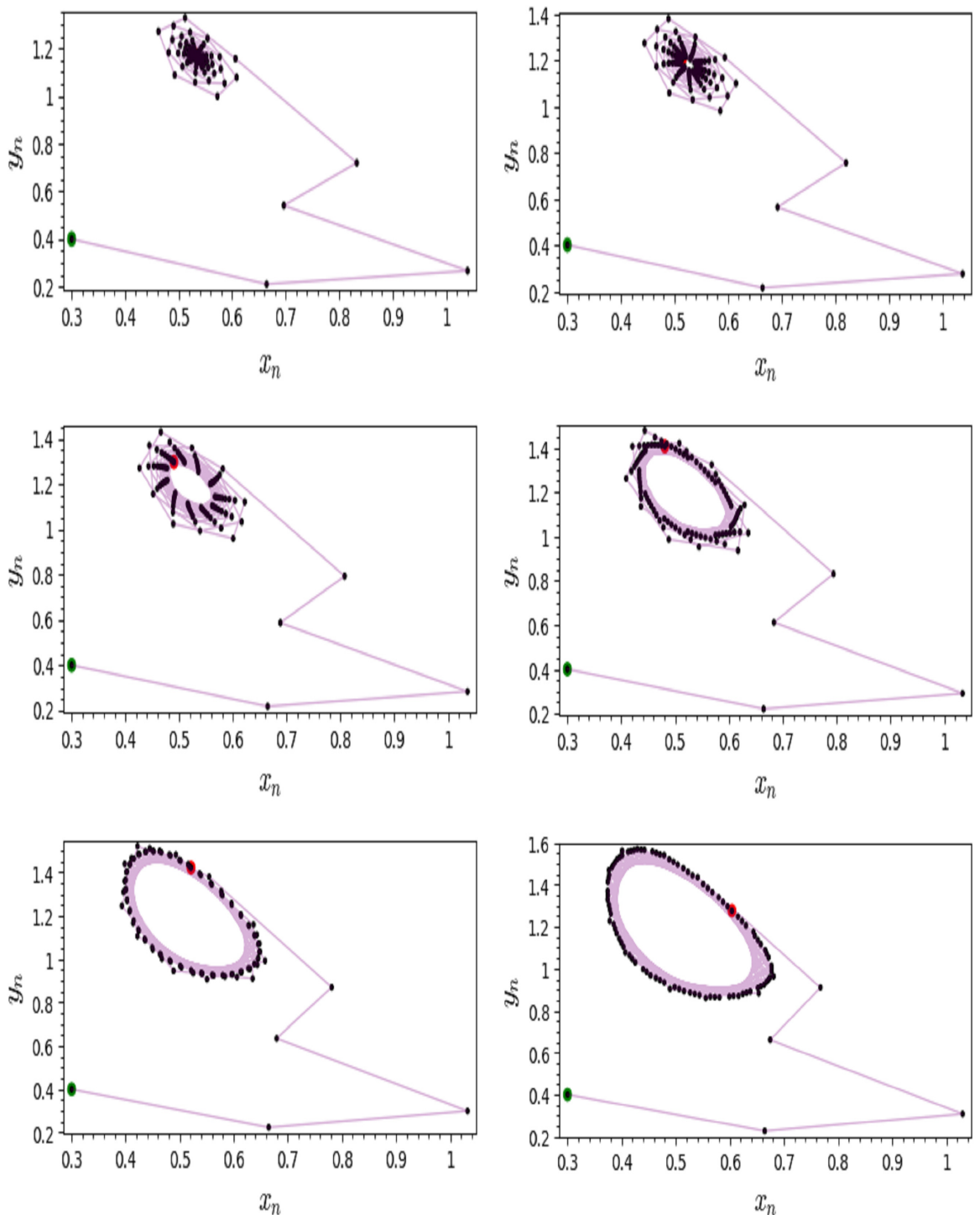

FIGURE 3. The phase portraits of the prey-predator system (1.2) for different values of $b$ with the parameter values $a=2.5, e=0.2, c=$ $1, H=1, h=0.9$ and the initial conditions $\left(x_{0}, y_{0}\right)=(0.3,0.4)$.

2.3111, the positive equilibrium points $(\bar{x}, \bar{y})$ are locally asymptotic stable and this equilibrium point $(\bar{x}, \bar{y})$ are unstable for $2.3111 \leq b \leq 3$. Furthermore, at the value 
$b=2.3111$, the positive equilibrium point $(\bar{x}, \bar{y})$ becomes unstable and also is produced closed invariant curve enclosing the unique positive unstable equilibrium point $(\bar{x}, \bar{y})$. So, Neimark Sacker bifurcation at $b=2.3111$ can be emerge. To clarify this situation, we obtain, $\lambda^{2}-0.837124 \lambda+1.00541=0$, the characteristic equation of Jacobian matrix evaluated at $(\bar{x}, \bar{y})=(0.519233,1.20192)$ with the parametric values $(e, a, c, H, h, b)=(0.2,2.5,1,1,0.9,2.3111)$. The eigenvalues of characteristic equation are $\lambda_{1,2}=0.415862 \mp 0.909422 i$ with $\left|\lambda_{1,2}\right|=1$. Consequently; we say that $(e, a, c, H, h, b)=(0.2,2.5,1,1,0.9,2.3111) \in N S B_{E_{2}}$

Example 4. In this example, we apply a state feedback control method by taking parameter values $(e, a, c, H, h, b)=(0.2,2.5,1,1,0.9,2.5)$ and initial condition $\left(x_{0}, y_{0}\right)=(0.3,0.4)$. Then corresponding controlled form is given by

$$
\begin{aligned}
& x_{n+1}=x_{n}+0.9\left(2.5 x_{n}\left(1-x_{n}-x_{n} y_{n}\right)-k_{1}\left(x_{n}-0.48\right)-k_{2}\left(y_{n}-1.3\right)\right. \\
& y_{n+1}=y_{n}+0.9\left[\left(2.5 x_{n} y_{n}-0.2 y_{n}-y_{n}\right] .\right.
\end{aligned}
$$

If marginal lines $l_{1}, l_{2}$ and $l_{3}$ in Eq.(4.5)-(4.7) are considered, we get

$$
\begin{aligned}
& l_{1}=-0.1836+k_{1}-2.925 k_{2}=0 \\
& l_{2}=3.1036-2 k_{1}+2.925 k_{2}=0
\end{aligned}
$$

and

$$
l_{3}=1.2636+2.925 k_{2}=0 .
$$

The marginal lines $l_{1}, l_{2}$ and $l_{3}$ determine the stable triangular region in the $k_{1} k_{2}$ plane. The region bounded by this lines of the controlled system (5.2) is plotted in Figure 4 .

\section{CONCLUSions}

This paper contains the complex dynamic behavior of a discrete-time the preypredator system (1.2). We investigate stability conditions of the equilibrium points of the system (1.2), and show that the system (1.2) displays a Neimark-Sacker bifurcations at coexistence equilibrium point. So, we apply the stabilizing feedback control methods to avoid chaos.

We find that the system (1.2) has a trivial (extinction) equilibrium $E_{0}$, an exclusion equilibrium $E_{1}$ and a coexistence equilibrium $E_{2}$. We get the asymptotic stability conditions of these equilibria by using the linearization method. It is clear that there is a unique positive coexistence equilibrium $E_{2}$ of the system (1.2) with $b>e+H$. Moreover, it is proved that the system (1.2) undergoes Neimark-Sacker bifurcation under the condition $b=\frac{1+e h+h H}{h}$ by using mathematical techniques of bifurcation theory. It is seen that the Neimark-Saker bifurcation appears when the parameters vary on the neighborhood

$$
N S B_{E_{2}}=\left\{a, b, e \in \mathbb{R}^{+}: b>\frac{e+H}{2}+\frac{1}{2} \sqrt{a e+e^{2}+a H+2 e H+H^{2}} \text { and } b=b_{1}\right\} .
$$




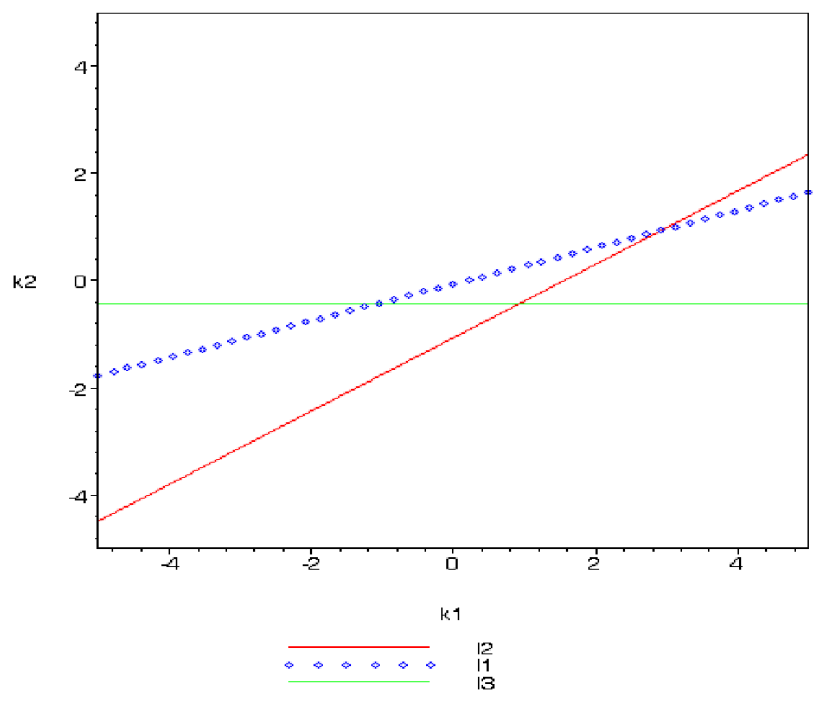

FIGURE 4. Stability region of the controlled system (5.2) in $k_{1} k_{2}$ plane

Some Figures present the dynamic properties of system (1.2) which has an chaos for $b>2.3111, e=0.2, a=2.5, c=1, H=1, h=0.9$ and the initial conditions $\left(x_{0}, y_{0}\right)=(0.3,0.4)$. We conclude that the parameter $b$ has a different effects on the dynamics of the system (1.2). Later, we obtain the conditions to control the chaos of the model via a state feedback method with the parameter $e=0.2, a=2.5, b=$ $2.5, c=1, H=1, h=0.9$. Chaos is successfully controlled by using control strategy.

\section{REFERENCES}

[1] P. Baydemir, H. Merdan, E. Karaoğlu, and G. Sucu, "Complex dynamics of a discrete-time prey predator system with leslie type: Stability bifurcation analysis and chaos," International Journal of Bifurcation and Chaos, vol. 30, no. 10, p. 2050149, 2020.

[2] S. Chakraborty, S. Pal, and N. Bairagi, "Predator-prey interaction with harvesting: Mathematical study with biological ramifications." Appl. Math. Model., vol. 36, no. 9, pp. 4044-4059, 2012, doi: 10.1016/j.apm.2011.11.029.

[3] C. W. Clark, Bioeconomic modelling and fisheries management. New York: Wiley, 1985.

[4] C. W. Clark, Mathematical bioeconomics: The optimal management of renewable resource. New York: Wiley, 1990.

[5] Q. Din, "Complexity and choas control in a discrete-time prey-predator model." Commun Nonlinear Sci. Numer. Simulat., vol. 49, pp. 113-134, 2017, doi: 10.1016/j.cnsns.2017.01.025.

[6] Q. Din, "Stability, bifurcation analysis and chaos control for a predator-prey system." J. Vib. Control, vol. 25, no. 3, pp. 612-626, 2018, doi: 10.1177/1077546318790871.

[7] Q. Din, O. A. Gümüs, and H. Khalil, "Neimark-Sacker bifurcation and chaotic behaviour of a modified host-parasitoid model." Z. Naturforsch. A, vol. 72, no. 1, pp. 25-37, 2017, doi: 10.1515/zna-2016-0335. 
[8] S. N. Elaydi, An introduction to difference equations. New York: Springer-Verlag, 1996. doi: 10.1007/0-387-27602-5.

[9] H. I. Freedman, Deterministic mathematical models in population ecology. New York: Marcel Dekker, 1980. doi: 10.2307/3556198.

[10] X. Gao, S. Ishag, S. Fu, W. Li, and W. Wang, "Bifurcation and Turing pattern formation in a diffusive ratio-dependent predator-prey model with predator harvesting." Nonlinear Analysis: Real World Applications, vol. 51, pp. 1-28, 2020, doi: 10.1016/j.nonrwa.2019.102962.

[11] B. S. Goh, Management and analysis of biological populations. Amsterdam, The Netherlands: Elsevier, 1980.

[12] J. Guckenheimer and P. J. Holmes, Nonlinear oscillations, dynamical systems, and bifurcations of vector fields. New York: Springer, 1983. doi: 10.1007/978-1-4612-1140-2.

[13] O. A. Gümüş and S. Acer, "Period-doubling bifurcation analysis and stability of epidemic model." Journal of Science and Arts, vol. 4, no. 49, pp. 905-914, 2019.

[14] O. A. Gümüş, A. G. M. Selvam, and D. A. Vianny, "Bifurcation and stability analysis of a discrete time SIR epidemic model with vaccination.” Int. J. Anal. Appl., vol. 17, no. 5, pp. 809-820, 2019, doi: 10.28924/2291-8639.

[15] Z. M. He and X. Lai, "Bifurcation and chaotic behavior of a discrete-time predator prey system." Nonlinear Anal. Real World Appl., vol. 12, pp. 403-417, 2011, doi: 10.1016/j.nonrwa.2010.06.026.

[16] C. M. Heggerud and K. Q. Lan, "Local stability analysis of ratio-dependent predator-prey models with predator harvesting rates." Appl. Math. Comput., vol. 270, pp. 349-357, 2015, doi: 10.1016/j.amc.2015.08.062.

[17] Z. Hu, Z. Teng, and L. Zhang, "Stability and bifurcation analysis of a discrete predator-prey model with nonmonotonic functional response." Nonlinear Anal. Real World Appl., vol. 12, no. 4, pp. 2356-2377, 2011, doi: 10.1016/j.nonrwa.2011.02.009.

[18] S. Kapçak, "Discrete dynamical systems with sage math." The Electronic Journal of Mathematics and Technology, vol. 12, no. 2, pp. 292-308, 2018.

[19] J. L. Kaplan and J. A. Yorke, "Preturbulence: a regime observed in a fluid flow model of Lorenz." Commun.Math. Phys., vol. 67, no. 2, pp. 93-108, 1979, doi: 10.1007/BF01221359.

[20] Y. A. Kuznetsov, Elements of applied bifurcation theory. New York: Springer-Verlag, 1998. doi: 10.1007/978-1-4757-3978-7.

[21] X. L. Liu and D. M. Xiao, "Bifurcation in a discrete time Lotka-Volterra predatorprey system.” Discrete Contin. Dyn. Syst. Ser. B, vol. 6, no. 3, pp. 559-572, 2006, doi: 10.3934/dcdsb.2006.6.559.

[22] X. L. Liu and D. M. Xiao, "Complex dynamic behaviors of discrete-time predator-prey system." Chaos Solitons Fract., vol. 32, no. 1, pp. 80-94, 2007, doi: 10.1016/j.chaos.2005.10.081.

[23] A. J. Lotka, Elements of physical biology. Williams and Wilkins Company, 1925.

[24] V. Madhusudanan, K. Anitha, S. Vijaya, and M. Gunasekaran, "Complex effects in discrete time prey-predator model with harvesting on prey." The International Journal of Engineering and Science, vol. 3 , no. 4, pp. 1-5, 2014.

[25] V. Madhusudanan, M. Gunasekaran, and S. Vijaya, "Diseased prey with harvesting predator in prey-predator system - an analytical study." IOSR Journal of Mathematics, vol. 9, no. 6, pp. 114-118, 2014, doi: 10.9790/5728-096114118.

[26] J. D. Murray, Mathematical biology. New York: Springer-Verlag, 1993. doi: 10.1007/b98868.

[27] E. Ott, C. Grebogi, and J. A. Yorke, "Controlling Chaos." Phys. Rev. Lett., vol. 64, no. 11, pp. 1196-1199, 1990, doi: 10.1103/PhysRevLett.64.1196.

[28] S. M. Rana, "Bifurcation and complex dynamics of a discrete-time predator-prey system." Computational Ecology and Software, vol. 5, no. 2, pp. 187-200, 2015. 
[29] S. M. S. Rana, "Bifurcation and complex dynamics of a discrete-time predator prey system with simplified Monod-Haldane functional response." Advances in Difference Equations, vol. 345, pp. 1-17, 2015, doi: 10.1186/s13662-015-0680-7.

[30] C. Robinson, Dynamical systems: stability, symbolic dynamics and chaos. Boca Raton: CRC Press, 1998. doi: 10.1201/9781482227871.

[31] F. J. Romeiras, C. Grebogi, E. Ott, and W. P. Dayawansa, "Controlling chaotic dynamical systems." Physica D: Nonlinear Phenomena, vol. 58, no. 1-4, pp. 165-192, 1992, doi: 10.1016/01672789(92)90107-X.

[32] M. Shahin, Explorations of mathematical models in biology with Maple. New Jersey: John Wiley\& Sons, Inc. Hoboken, 2015.

[33] V. Volterra, "Variations and fluctuations in the numbers of coexisting animal species." Lecture notes in Biomathematics, vol. 22, pp. 1923-1940, 1927.

[34] S. A. Wuhaib and Y. Abu Hasan, "Predator-prey interactions with harvesting of predator with prey refuge." Commun. Math. Biol. Neurosci., vol. 2013, no. 1, pp. 1-19, 2013.

[35] D. Xiao and L. S. Jennings, "Bifurcations of a ratio-dependent predator-prey system with constant rate harvesting." SIAM J. Appl. Math., vol. 65, no. 3, pp. 737-753, 2005, doi: 10.1137/S0036139903428719.

[36] D. Xiao, W. Li, and M. Han, "Dynamics in a ratio-dependent predator-prey model with predator harvesting." J. Math. Anal. Appl, vol. 324, pp. 14-29, 2006, doi: 10.1016/j.jmaa.2005.11.048.

[37] L. G. Yuan and Q. G. Yang, "Bifurcation, invariant curve and hybrid control in a discrete-time predator-prey system." Applied Mathematical Modeling, vol. 39, no. 8, pp. 2345-2362, 2015, doi: 10.1016/j.apm.2014.10.040.

[38] M. Zhao, C. Li, and J. Wang, "Complex dynamic behaviors of a discrete-time predatorprey system." Journal of Applied Analysis and Computation, vol. 7, pp. 478-500, 2017, doi: 10.11948/2017030.

Authors' addresses

Özlem Ak Gümüiş

(Corresponding author) Adiyaman University, Faculty of Arts and Sciences, Department of Mathematics, 02040, Adiyaman, Turkey.

E-mail address: akgumus@adiyaman.edu.tr

Michal Fečkan

Mathematical Institute, Slovak Academy of Sciences, Štefánikova 49, 81473 Bratislava, Slovakia. Department of Mathematical Analysis and Numerical Mathematics,, Comenius University in Bratislava,, Mlynská dolina, 84248 Bratislava, Slovakia

E-mail address: michal. feckan@gmail.com 